\title{
Probabilistic modeling of the training test subdivision of device production
}

\author{
A. L. Litvinov \\ O.M. Beketov National Universityof Urban Economy in Kharkov, Ukraine \\ Corresponding author. E-mail: litan6996@gmail.com
}

Paper received 05.12.20; Accepted for publication 18.12.20.

\author{
https://doi.org/10.31174/SEND-NT2021-250IX31-09
}

Abstract. The paper deals with the probabilistic modeling of training tests subdivision of device production. Increased reliability is required from devices for control systems of non-stationary objects. This is provided by a number of training operations with subsequent control. A network model of queuing systems is proposed to describe the process of passing devices through subdivision of control-training operations. Expressions are obtained, which allow estimating the load of each production section and find its characteristics of functioning.

Keywords: modeling, training operations, network, queuing system, probability.

Introduction. Control systems for non-stationary objects (cars, airplanes, ships) include a significant number of various devices, such as information sensors, controllers and computers. As a rule, they function in conditions of an aggressive external environment. The temperature can vary from $-120^{\circ} \mathrm{C}$ to $+50^{\circ} \mathrm{C}$, devices are affected with vibration during movement, high humidity can lead to loss of dielectric properties of materials. At the same time, high requirements are imposed on the reliability of the operation of control systems for non-stationary objects. It is possible to provide high reliability of such objects both at the design stage and at the stage of production, by including training operations of devices in appropriate conditions on special stands with subsequent monitoring for correct functioning [1]. Thus, the simplified technological scheme of the training tests subdivision of device production can be the same as in fig. 1 .

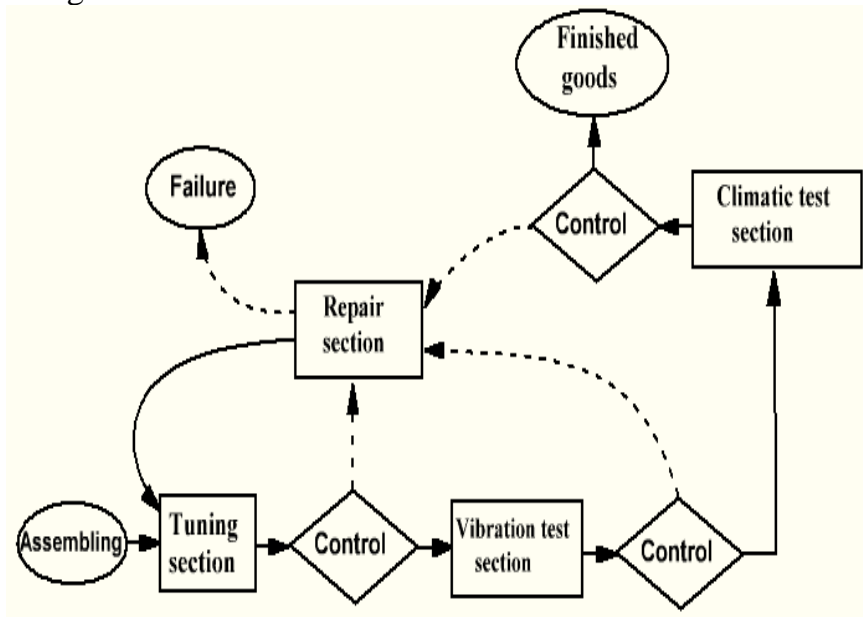

Figure 1 - Technological scheme of the training tests subdivision of device production

Failures of devices during training operations are random. This leads to congestion of individual technological sections and transport conveyors, reducing the capacity and production capacity of the entire system. At the same time, as practice shows, such production systems designed according to well-developed methods of flow-mass production design often can not provide the required productivity and throughput, despite the sufficient capacity of the assembly sections. Possible irregularity of production also render a strong influence on the performance of the system. In this regard, the traditional methods of calculation of the flow-mass production design of devices production must be supplemented by probabilistic methods that allow take into account various random factors affecting the production process.

In a formalized presentation, each technological section of the training test subdivision is a service system consisting of a certain number of service places (these may be the workplaces of the regulators or the seats in the climatic test area), the input flow of devices that act as requests for service, and the place of waiting for requests, in whose role the intermediate buffers can be used. In the whole, the training tests subdivision of device production can be modeling by a queuing network, in which the assembly section is the source of requests (in this case, the assembled devices), separate technological sections are separate service systems.

A brief overview of publications on the topic. A characteristic feature of the modern stage of development of society is the increasingly widespread use of large complex systems with a network structure and stochastic nature of functioning. The study of the processes occurring in systems of this kind is carried out within the framework of the theory of networks of queues and simulation. The basic work on queueing networks did James Jackson (University of California, Los Angeles, mathematics professor) [2]. The work [3] is devoted to the study of closed homogeneous exponential queueing networks. To analyze the quantitative distribution of customers over the nodes of the queueing network, a diffusion approximation method was proposed in [4]. Along with analytical methods, simulation methods are widely used to study queueing networks [5, 6]. Simulation modeling allows obtain more accurate results than analytical methods, but simulation models have a little typification, in addition, the cost of developing simulation models is quite high. The applied aspects of queueing networks, as applied to computer systems, are described in [7, 8]. It should be noted that most of the works on the applied use of queueing networks are devoted to computer systems and networks. Taking into account the specifics of production processes building appropriate models of queueing networks is an urgent task.

Purpose of research. The probabilistic model of the training tests subdivision of device production will be a queuing network with an appropriate topology. The aim of the work is to determine, based on the queuing network, the load of each production section, the average 
cycle time of control and training tests, the effect of device failures on the general characteristics of the system.

Materials and methods. The structure of the training tests subdivision of device production can be represented by the network transmission graph, shown in fig. 2. The node $\mathrm{S}_{0}$ corresponds to the assembly section, $\mathrm{S}_{1}-$ to the tuning section, $S_{2}-$ to the vibration test section, $S_{3}-$ to the climatic test section, $\mathrm{S}_{4}-$ to the repair section. Solid arcs correspond to the passage of devices along the technological chain without failures; arcs marked with strokes mean the transfer of failed devices to the repair section, or a completely defective product. $p_{i j}$ means the probability of transferring the devices from section $i$ to section $\mathrm{j}$. Accordingly, $p_{23}$ is the probability that the device has passed the vibration test section normally and $p_{24}$ is the probability that the device require a repair after vibration test, $p_{30}$ is the probability that the device after section of climatic tests has passed all the training tests normally and $p_{34}$ is the probability that the device require a repair after climatic test, $p_{40}-$ this is the probability that the device is not subject to recovery.

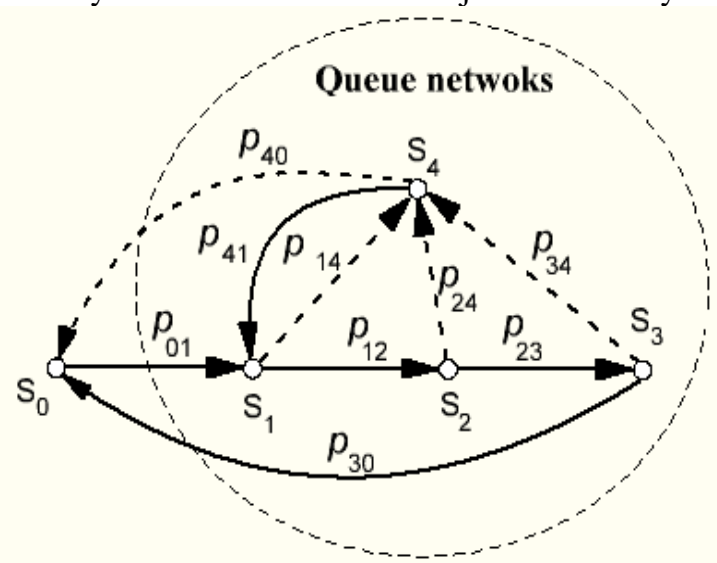

Figure 2 - Networks transmission graph

Let us define the transition probability matrix $P$ as consisting of elements $p_{i j}$, that is

$P=\left[\begin{array}{ccccc}0 & 1 & 0 & 0 & 0 \\ 0 & 0 & p_{12} & 0 & p_{14} \\ 0 & 0 & 0 & p_{23} & p_{24} \\ p_{30} & 0 & 0 & 0 & p_{34} \\ p_{40} & p_{41} & 0 & 0 & 0\end{array}\right]$.

The peculiarity of this matrix is that the sum of the probabilities in the rows is equal to one.

We will investigate the functioning of the system in a stationary state. Let us denote by $\lambda_{i}$ the total intensity of the input flow of devices in section $i$ in a stationary mode, $\tau_{i}-$ the average time of the technological operation in the $i$-th section. For the existence of a stationary state, it is necessary that the inequality $\rho_{i}=\lambda_{i} \tau_{i}<1$ holds for each section, where $\rho_{i}$ is a load of $i$-th section. Then the following system of equations for the stationary state corresponds to the graph in fig. 2 $-\lambda_{0}+p_{30} \cdot \lambda_{3}+p_{40} \cdot \lambda_{4}=0$

$\lambda_{0}-\lambda_{1}+p_{41} \cdot \lambda_{4}=0$,

$p_{12} \cdot \lambda_{1}-\lambda_{2}=0$,

$p_{23} \cdot \lambda_{2}-\lambda_{3}=0$,

$p_{14} \cdot \lambda_{1}+p_{24} \cdot \lambda_{2}+p_{34} \cdot \lambda_{3}-\lambda_{4}=0$.

In the matrix form, the system of equations (2) is written by

$$
\left[\begin{array}{ccccc}
-1 & 0 & 0 & p_{30} & p_{40} \\
1 & -1 & 0 & 0 & p_{41} \\
0 & p_{12} & -1 & 0 & 0 \\
0 & 0 & p_{30} & -1 & 0 \\
0 & p_{14} & p_{24} & p_{34} & -1
\end{array}\right] \cdot\left[\begin{array}{c}
\lambda_{0} \\
\lambda_{1} \\
\lambda_{2} \\
\lambda_{3} \\
\lambda_{4}
\end{array}\right]=\left[\begin{array}{c}
0 \\
0 \\
0 \\
0 \\
0
\end{array}\right] .
$$

If we add to the elements of the last row in system matrix corresponding elements of all the remaining rows, we get a row with zero elements. Thus, the determinant of the system matrix of the system of equations (3) is equal zero. That is, the system of equations is uncertain. But if we fix the intensity of the arrival of devices from the assembly section $\lambda_{0}$, then the system of equations becomes definite and can be solved relatively $\lambda_{0}$, that can be expressed as

$$
\lambda_{i}=\alpha_{i} \lambda_{0}, i=1,2,3,4 .
$$

Let us reformat the system of equations (2) as follows

$$
\begin{array}{rlrl} 
& p_{30} \cdot \lambda_{3}+p_{40} \cdot \lambda_{4} & =\lambda_{0}, \\
-\lambda_{1} & & +p_{41} \cdot \lambda_{4} & =-\lambda_{0}, \\
p_{12} \cdot \lambda_{1}-\lambda_{2} & & =, \\
p_{23} \cdot \lambda_{2}-\lambda_{3} & =0, \\
p_{14} \cdot \lambda_{1}+p_{24} \cdot \lambda_{2}+p_{34} \cdot \lambda_{3} & -\lambda_{4}=0 .
\end{array}
$$

Reordering the corresponding rows, rewrite the system of equations (5) as follows:

$$
\begin{array}{rlrl}
-1 \cdot \lambda_{1} & +p_{41} \cdot \lambda_{4} & =-1 \cdot \lambda_{0}, \\
p_{12} \cdot \lambda_{1}-1 \cdot \lambda_{2} & =0, \\
p_{14} \cdot \lambda_{1}+p_{24} \cdot \lambda_{2}+p_{34} \cdot \lambda_{3}-1 \cdot \lambda_{4} & =0, \\
p_{23} \cdot \lambda_{2}-1 \cdot \lambda_{3} & =0, \\
p_{30} \cdot \lambda_{3}+p_{40} \cdot \lambda_{4} & =1 \cdot \lambda_{0} .
\end{array}
$$

The solution of the system of linear algebraic equations (6) is expediently sought by the Gauss method in a matrix form, which makes it possible to simultaneously test the system for solvability. We write the extended matrix system (6) in augmented matrix form (only coefficients for unknowns) as follows

$$
\begin{array}{cccc|l}
-1 & 0 & 0 & p_{41} & -1 \\
p_{12} & -1 & 0 & 0 & 0 \\
p_{14} & p_{24} & p_{34} & -1 & 0 \\
0 & p_{23} & -1 & 0 & 0 \\
0 & 0 & p_{30} & p_{40} & 1
\end{array} .
$$

Let us realize the direct move of the Gauss method. To do this, multiply all the elements of the first row by $p_{12}$ and add them to the corresponding elements of the second row. After that, multiply all elements of the first row by $p_{14}$ and add them to the corresponding elements of the third row. These operations are formally written as follows. 


$$
\left\{I I:=I I+I \cdot \Pi_{12}, I I I:=I I I+I \cdot p_{14}\right\}
$$

As a result, we get

$$
\begin{array}{cccc|c}
-1 & 0 & 0 & p_{41} & -1 \\
0 & -1 & 0 & p_{41} p_{12} & -p_{12} \\
0 & p_{24} & p_{34} & p_{41} p_{14}-1 & p_{14} \\
0 & p_{23} & -1 & 0 & 0 \\
0 & 0 & p_{30} & p_{40} & 1
\end{array}
$$

Next, we perform the following operations

$$
\left\{I I I:=I I I+I I \cdot p_{24}, I V:=I V+I I \cdot p_{23}\right\}
$$

As a result, we get

$$
\begin{array}{cccc|c}
-1 & 0 & 0 & p_{41} & -1 \\
0 & -1 & 0 & p_{41} p_{12} & -p_{12} \\
0 & 0 & p_{34} & p_{41} p_{14}+p_{41} p_{12} p_{24}-1 & -p_{14}-p_{12} p_{24} \\
0 & 0 & -1 & p_{41} p_{12} p_{23} & -p_{12} p_{23} \\
0 & 0 & p_{30} & p_{40} & 1
\end{array}
$$

Perform the following operation: $\{V:=V+I I I\}$

As a result, we get

$$
\begin{array}{cccc|c}
-1 & 0 & 0 & p_{41} & -1 \\
0 & -1 & 0 & p_{41} p_{12} & -p_{12} \\
0 & 0 & p_{34} & p_{41} p_{14}+p_{41} p_{12} p_{24}-1 & -p_{14}-p_{12} p_{24} \\
0 & 0 & -1 & p_{41} p_{12} p_{23} & -p_{12} p_{23} \\
0 & 0 & p_{30}+p_{34} & p_{40}+p_{41} p_{14}+p_{41} p_{12} p_{24}-1 & 1-p_{14}-p_{12} p_{24}
\end{array}
$$

Above the elements of the fifth row perform the following transformations:

$$
\begin{aligned}
& p_{30}+p_{34}=1 \\
& p_{40}+p_{41} p_{14}+p_{41} p_{12} p_{24}-1=-\left(1-p_{40}\right)+p_{41} p_{14}+p_{41} p_{12} p_{24}= \\
& =-p_{41}+p_{41} p_{14}+p_{41} p_{12} p_{24}=-p_{41}\left(1-p_{14}\right)+p_{41} p_{12} p_{24}= \\
& =-p_{41} p_{12}+p_{41} p_{12} p_{24}=-p_{41} p_{12}\left(1-p_{24}\right)=-p_{41} p_{12} p_{23} \\
& 1-p_{14}-p_{12} p_{24}=\left(1-p_{14}\right)-p_{12} p_{24}=p_{12}-p_{12} p_{24}= \\
& =p_{12}\left(1-p_{24}\right)=p_{12} p_{23} .
\end{aligned}
$$

As a result, we get

$$
\begin{array}{cccc|c}
-1 & 0 & 0 & p_{41} & -1 \\
0 & -1 & 0 & p_{41} p_{12} & -p_{12} \\
0 & 0 & p_{34} & p_{41} p_{14}+p_{41} p_{12} p_{24}-1 & -p_{14}-p_{12} p_{24} \\
0 & 0 & -1 & p_{41} p_{12} p_{23} & -p_{12} p_{23} \\
0 & 0 & 1 & -p_{41} p_{12} p_{23} & p_{12} p_{23}
\end{array} .
$$

The fourth and fifth rows of the system (7) are equivalent. Throw away the fifth row and swap the third and fourth rows. We get

$$
\begin{array}{cccc|c}
-1 & 0 & 0 & p_{41} & -1 \\
0 & -1 & 0 & p_{41} p_{12} & -p_{12} \\
0 & 0 & -1 & p_{41} p_{12} p_{23} & -p_{12} p_{23} \\
0 & 0 & p_{34} & p_{41} p_{14}+p_{41} p_{12} p_{24}-1 & -p_{14}-p_{12} p_{24}
\end{array}
$$

Using the third row, let us perform the following conversion: $\left\{I V:=I V+I I I \cdot p_{34}\right\}$

We get

$$
\begin{array}{ccc}
-1 & 0 & 0 \\
0 & -1 & 0 \\
0 & 0 & -1 \\
0 & 0 & 0
\end{array}
$$$$
p_{41}
$$$$
p_{41} p_{12}
$$$$
p_{41} p_{12} p_{23}
$$

$$
\begin{aligned}
& -1 \\
& -p_{12} \\
& -p_{12} p_{23} \\
& p_{41} p_{14}+p_{41} p_{12} p_{24}+p_{41} p_{12} p_{23} p_{34}-1-p_{14}-p_{12} p_{24}-p_{12} p_{23} p_{34}
\end{aligned}
$$

From the last row follows:

$$
\lambda_{4}=\frac{p_{14}+p_{12} p_{24}+p_{12} p_{23} p_{34}}{1-p_{41} p_{14}-p_{41} p_{12} p_{24}-p_{41} p_{12} p_{23} p_{34}} \lambda_{0} .
$$

Accordingly, from the third row we get:

$$
\lambda_{3}=p_{12} p_{23} \cdot \lambda_{0}+p_{41} p_{12} p_{23} \lambda_{4}
$$

and from the first row we get

$$
\lambda_{1}=\lambda_{0}+p_{41} \cdot \lambda_{4}
$$

So

$$
\lambda_{3}=p_{12} p_{23} \cdot \lambda_{0}+p_{41} p_{12} p_{23} \lambda_{4}
$$

from the second row we get

$$
\begin{aligned}
& \alpha_{4}=\frac{p_{14}+p_{12} p_{24}+p_{12} p_{23} p_{34}}{1-p_{41} p_{14}-p_{41} p_{12} p_{24}-p_{41} p_{12} p_{23} p_{34}}, \alpha_{3}=p_{12} p_{23}+p_{41} p_{12} p_{23} \alpha_{4}, \\
& \alpha_{2}=p_{12}+p_{41} p_{12} \alpha_{4}, \alpha_{1}=1+p_{41} \alpha_{4} .
\end{aligned}
$$

The most important indicators of production will be the intensity of devices that have completely passed the training tests $\lambda_{\text {out }}$ and the intensity of completely defective devices $\lambda_{\text {fail }}$. From the network transmission graph follows:

$$
\lambda_{\text {out }}=\lambda_{3} p_{30}, \lambda_{\text {fail }}=\lambda_{4} p_{40} \text {. }
$$

Important characteristic of the production process is the average time of the devices passing through the training and control operations $-T$. It, in turn, depends on the average time spent by devices (customers in terms of queuing theory) in each section $t_{i}, i=1,2,3,4$. Then

$$
T=\sum_{i=1}^{4} \alpha_{i} t_{i}[9]
$$

The execution time of a production operation in the $i$-th section is constant $-\tau_{i}$. Therefore, a queuing system of the type $\mathrm{M} / \mathrm{D} / 1$ can be chosen as a model of production section. Then $t_{i}=\frac{\rho_{i}}{2\left(1-\rho_{i}\right)}+\frac{1}{\mu_{i}} \quad$ [10]. After transformations

$t_{i}=\frac{\rho_{i}}{2\left(1-\rho_{i}\right)}+\frac{1}{\mu_{i}}=\frac{\lambda_{i} \tau_{i}}{2\left(1-\lambda_{i} \tau_{i}\right)}+\tau_{i}=\left[\frac{\alpha_{i} \lambda_{0}}{2\left(1-\alpha_{i} \lambda_{0} \tau_{i}\right)}+1\right] \tau_{i} \quad$ and $T=\sum_{i=1}^{4}\left[\frac{\alpha_{i} \lambda_{0}}{2\left(1-\alpha_{i} \lambda_{0} \tau_{i}\right)}+1\right] \tau_{i} \alpha_{i}$

By using obtained expressions the performance of the training test sections can be reasonably chosen.

Results and their discussion. Let the transition probability matrix $\boldsymbol{P}$ of the network have the form: 


$$
P=\left[\begin{array}{ccccc}
0 & 1 & 0 & 0 & 0 \\
0 & 0 & 0,8 & 0 & 0,2 \\
0 & 0 & 0 & 0,9 & 0,1 \\
0,7 & 0 & 0 & 0 & 0,3 \\
0,3 & 0,7 & 0 & 0 & 0
\end{array}\right]
$$

Using the expressions (8), we get:

$$
\begin{gathered}
\lambda_{1}=1,53 \lambda_{0}, \lambda_{2}=1,23 \lambda_{0}, \lambda_{3}=1,10 \lambda_{0}, \lambda_{4}=0,76 \lambda_{0} . \\
\text { Accordingly, } \lambda_{\text {out }}=0,77 \lambda_{0}, \lambda_{\text {fail }}=0,76 \lambda_{0}
\end{gathered}
$$

Thus, the received expressions allow to estimate loading of each technological section and to choose its productivity.

On the fig. 3 depicted the family of curves $\lambda_{\text {out }}$ as a function of the probability of a complete failure of the device $p_{40}$ for different values of the probability of failures in the vibration test section - $p_{24}$.

The dependencies are almost linear, and each section contributes to the decrease in the performance of the entire system of training operations.

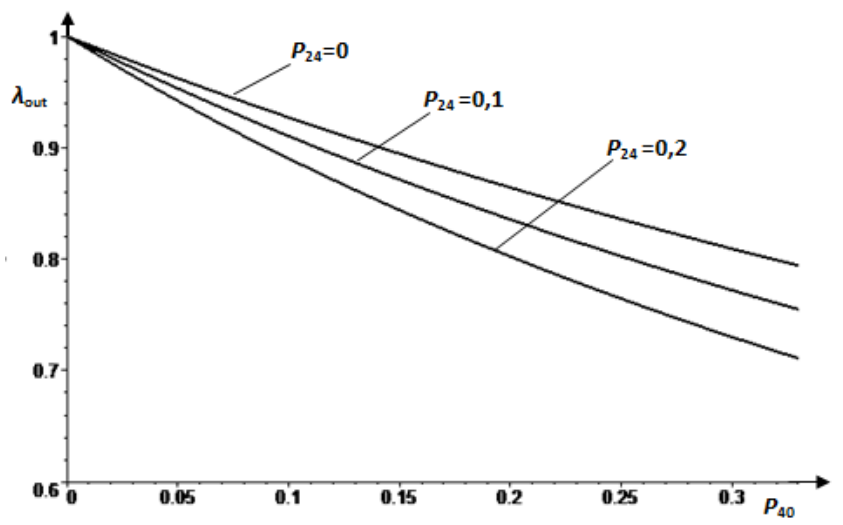

Figure 3. Related intensity of devices that have completely passed the training tests

Conclusions. The paper proposes the use of a queuing network as a model of the training tests subdivision of device production. The analytical solution of the corresponding systems of equations makes it possible to estimate the load of each training test section, the real time of the production cycle, the output of suitable devices from the section. By using obtained expressions the performance of the training test sections can be reasonably chosen.

\section{ЛИТЕРАТУРА}

1. Тупик В.А. Технология и организация производства радиоэлектронной аппаратуры. СПб.: Изд-во СПбГЭТУ «ЛЭТИ», 2004. - 144 c.

2. Jackson J.R. Networks of waiting lines //Operational Research, 1957, 5, №4. P. 518-521.

3. Gordon W.J., Newell G.F. Closed queeing systems with exponential servers // Operational Research, 1967, 15 № 2. P. 522529.

4. Kobayashi H. Application of diffusion approximation to queueing networks // Journal of the Association for Computing Machinery, 1974, 21 №2. P. 316-328.

5. Shannon R. System simulation. The art and science. Englewood Cliffs, new Jersey:Prentice Hall PTR, 1975. 418 p.

6. Banks J., Carson J. S., Nelson B. L., Nicol D. M. Discreteevent system simulation. 5th ed. Upper Saddle River, NJ : Prentice Hall, 2010. 622 p.

7. Kleinrock L. Queueing systems - Vol. 2: Computer applications. John Wiley \& Sons, 1976. 576 p.

8. Raj J. The Art of Computer systems performance analysis: Techniques for experimental design, measurement, simulation, and modeling. Wiley India Pvt. Limited, 2008. $716 \mathrm{p}$

9. Kaufmann A., Cruon R. Les phenomenes d'attente. Theorie et application. Paris: Dunod, 1961. - 302 p.

10. Соколов А. Н., Соколов Н. А. Однолинейные системы массового обслуживания. СПб.: Изд-во «Теледом», 2010. $-112 \mathrm{c}$.

\section{REFERENCES}

1. Tupik V.A. Technology and organization of production of radio-electronic equipment. St. Petersburg: Publishing house of SPETU "LETI", 2004. - 144 p.

10. Sokolov A.N., Sokolov N.A. Single-server queeing systems. St. Petersburg: Publishing house of "Teledom", 2010. - 112 p. 